\title{
Cancer-related long noncoding RNAs show aberrant expression profiles and competing endogenous RNA potential in esophageal adenocarcinoma
}

\author{
YANG YU*, XINGXING CHEN* and SHUNDONG CANG \\ Department of Oncology, Henan Provincial People's Hospital, People's Hospital of Zhengzhou University, \\ Zhengzhou University, Zhengzhou, Henan 450003, P.R. China
}

Received January 24, 2019; Accepted July 17, 2019

DOI: $10.3892 / \mathrm{ol} .2019 .10808$

\begin{abstract}
Long non-coding RNAs (lncRNAs) govern gene expression by competitively binding to microRNA response elements (MREs). Although they were initially considered as transcriptional noise, lncRNAs have attracted increased attention in oncology. Dysregulation of lncRNAs occurs in various types of human tumor, including esophageal adenocarcinoma (EAC). However, the functions of these cancer-associated IncRNAs and of their related competitive endogenous RNA (ceRNA) network in EAC remains unknown. To determine the relevant potential mechanisms, the present study analyzed the transcriptome sequencing data and clinical information of 79 patients with EAC, including 79 tumor samples and 11 normal samples, which were obtained from The Cancer Genome Atlas esophageal cancer project. The edgeR v3.25.0 software was used for differential gene expression analysis. The results exhibited 561 cancer-associated lncRNAs with a $>2.0$-fold change and a false discovery rate-adjusted $\mathrm{P}<0.01$. Among these lncRNAs, 26 were significantly associated with patient overall survival. According to data from bioinformatics databases and differentially expressed RNAs, an lncRNA-regulated ceRNA network for EAC was constructed. The results demonstrated that the aberrantly expressed lncRNA-associated ceRNA network included 37 EAC cancer-associated lncRNAs, five miRNAs and 13 mRNAs. In conclusion, the present study identified novel IncRNAs as candidate prognostic biomarkers and revealed a potential regulatory network of gene expression in EAC.
\end{abstract}

Correspondence to: Professor Shundong Cang, Department of Oncology, Henan Provincial People's Hospital, People's Hospital of Zhengzhou University, Zhengzhou University, 7 Weiwu Road, Zhengzhou, Henan 450003, P.R. China

E-mail: cangsdsy@126.com

*Contributed equally

Key words: esophageal adenocarcinoma, long non-coding RNA, competing endogenous RNA, The Cancer Genome Atlas, prognosis

\section{Introduction}

Esophageal adenocarcinoma (EAC) is a highly lethal malignancy that occurs mainly in the distal esophagus and gastroesophageal junction (1). EAC is rare in China; however, it represents the predominant type of esophageal cancer in North America and Europe. In these continents, the overall incidence of EAC has rapidly increased over the past three decades at a rate $(5-10 \%)$ greater than that of any other major cancer and the incidence rate is higher in white males compared with that in white females (2-4). The reason for this increase is not entirely understood. Previous studies have reported that EAC differs from esophageal squamous cell carcinoma (ESCC) in terms of genetic and environmental risk factors such as tobacco use, alcohol, obesity and germline mutations $(5,6)$. Systematic therapy for EAC typically includes endoscopic mucosal resection, surgical resection, chemoradiotherapy and neoadjuvant chemotherapy; however, the mortality rate remains high and the overall 5-year survival rate is $17 \%$ in the United States (7). Although endoscopy can accurately diagnose early-stage EAC, most patients are diagnosed with regional metastasis or distant metastasis, which are positively correlated with a considerable decline in the 5 -year survival rate (8). There is therefore an urgency to identify novel potential diagnostic and prognostic biomarkers for EAC.

Long non-coding RNAs (lncRNAs) represent a new class of non-coding RNAs (ncRNAs) and are defined as transcripts $>200$ nucleotides in length (9). Unlike their shorter counterparts, including microRNAs (miRNAs), the roles and underlying mechanisms of lncRNAs in human disease remain largely unknown. Due to improvements in DNA sequencing techniques, numerous IncRNAs have been discovered. In addition, an increasing number of lncRNAs have been identified in human cancer, such as HAGLR opposite strand lncRNA overexpression in gastric cancer (10). Previous studies have focused on the biological function and underlying molecular mechanism of lncRNAs in various types of cancer, including colorectal cancer, gastric cancer, hepatocellular carcinoma, renal cell carcinoma, prostate carcinoma and EAC (11-17). Although studies reported that lncRNAs can be involved in the development and progression of $\operatorname{ESCC}(6,14)$, only 
a few studies have determined the function of lncRNAs in $\operatorname{EAC}(5,18)$.

Emerging technologies have increased our ability to determine the functions of cancer-associated lncRNAs. Significant progress towards understanding the underlying molecular mechanism by which IncRNAs can regulate miRNA function has therefore been made. Salmena et al (19) proposed a competing endogenous RNA (ceRNA) language where protein coding genes, microRNAs and lncRNAs communicate with each other by competitively binding to shared miRNA response elements (MREs). Competing endogenous RNA networks comprise a new regulatory network of mRNAs and non-coding RNAs, which reveals a greatly expanded role for IncRNAs in human disease (20). This hypothesis has been experimentally validated. For example, Cesana et al (21) identified a muscle-specific lncRNA named linc-MD1, which regulates the expression of mastermind-like 1 and myocyte-specific enhancer factor $2 \mathrm{C}$ by serving as a 'sponge' for miR-133. Furthermore, Qu et al (22) demonstrated that lncARSR mediates sunitinib resistance in renal cell carcinoma by competitively binding to miR-34/miR-449 to promote AXL receptor tyrosine kinase and c-MET expression. Exploration of RNA cross-talk offers therefore insights into cancer diagnosis and therapy. An lncRNA-miRNA-mRNA ceRNA network has therefore been constructed for various types of human cancer, in particular for ESCC (23-26); however, such a network has not yet been described for EAC.

In order to systematically describe EAC-associated pseudogenes and to construct a ceRNA network, the present study comprehensively analyzed RNA sequencing (RNA-Seq) transcript data that were obtained from The Cancer Genome Atlas (TCGA) esophageal cancer project (https://www.cancer. gov/types/esophageal). The database includes lncRNA, microRNA and mRNA data and clinical information from patients with EAC. The present study included 79 EAC tumor and 11 adjacent non-tumor esophagus tissue samples. By using publicly available RNA-Seq data from TCGA, some EAC-associated lncRNAs, mRNAs and miRNAs were identified based on the ceRNA hypothesis. Furthermore, 561 differentially expressed lncRNAs (DElncRNAs), 1,289 differentially-expressed mRNAs (DEmRNAs) and 44 differentially-expressed miRNAs (DEmiRNAs) were identified. Subsequently, five dysregulated lncRNAs, 13 mRNAs and 32 miRNAs were identified and included in a constructed ceRNA network based on IncRNA-miRNA interactions predicted by miRcode v11 (www.mircode.org/). Potential prognostic biomarkers were then identified by exploring the influence of dysregulated RNAs on overall survival using the univariate Cox proportional hazards regression model and Kaplan-Meier curve analysis. The results from this comprehensive analysis provided the foundation for deeper understanding of the cancer-associated lncRNA functions in EAC and revealed potential prognostic biomarkers.

\section{Materials and methods}

Patients and samples. Data for 187 patients with esophageal cancer were obtained from the TCGA data portal (https://portal.gdc.cancer.gov/). The exclusion criteria were as follows: i) Patients with ESCC or undetermined pathological
Table I. Clinical characteristics of patients with esophageal adenocarcinoma.

\begin{tabular}{|c|c|c|}
\hline Characteristics & No. cases & $\%$ \\
\hline \multicolumn{3}{|c|}{ Age at diagnosis, years } \\
\hline$<60$ & 26 & 32.9 \\
\hline$\geq 60$ & 53 & 67.1 \\
\hline \multicolumn{3}{|l|}{ Sex } \\
\hline Male & 11 & 13.9 \\
\hline Female & 68 & 86.1 \\
\hline \multicolumn{3}{|l|}{ Metastasis } \\
\hline M0 & 57 & 72.2 \\
\hline M1 & 10 & 12.7 \\
\hline MX & 10 & 12.7 \\
\hline \multicolumn{3}{|c|}{ Lymph node status } \\
\hline NO & 22 & 27.8 \\
\hline N1 & 44 & 55.7 \\
\hline $\mathrm{N} 2$ & 5 & 6.3 \\
\hline N3 & 5 & 6.3 \\
\hline NX & 3 & 3.8 \\
\hline \multicolumn{3}{|l|}{ Stage } \\
\hline I & 9 & 11.4 \\
\hline II & 22 & 27.8 \\
\hline III & 26 & 32.9 \\
\hline IV & 5 & 6.3 \\
\hline \multicolumn{3}{|l|}{ T stage } \\
\hline T0 & 1 & 1.3 \\
\hline $\mathrm{T} 1$ & 19 & 24.1 \\
\hline $\mathrm{T} 2$ & 10 & 12.7 \\
\hline $\mathrm{T} 3$ & 46 & 58.2 \\
\hline $\mathrm{T} 4$ & 1 & 1.3 \\
\hline $\mathrm{TX}$ & 2 & 2.6 \\
\hline \multicolumn{3}{|c|}{ Histological grade } \\
\hline G1 & 1 & 1.3 \\
\hline $\mathrm{G} 2$ & 28 & 35.4 \\
\hline G3 & 24 & 30.4 \\
\hline GX & 26 & 32.9 \\
\hline
\end{tabular}

classification; and ii) samples without corresponding RNA-Seq and miRNA-Seq data. Overall, data from 79 patients with EAC were enrolled in the present study. This study followed the publication guidelines provided by TCGA (http://cancergenome.nih. gov/publications/publicationguidelines).

RNA-Seq data. RNA-Seq and miRNA-Seq data (level 3) were downloaded from 90 tissue samples of the TCGA database, including 79 EAC samples and 11 adjacent normal samples. The gene expression profiles generated from Illumina Hiseq platforms (Illumina, Inc.) were all publicly available data.

Analysis of DEmRNAs, DElcnRNAs and DEmiRNAs. The raw count data were processed with edgeR v3.25.0 (Bioconductor), which is a package based on the R language (v3.5.0) (27) 
A

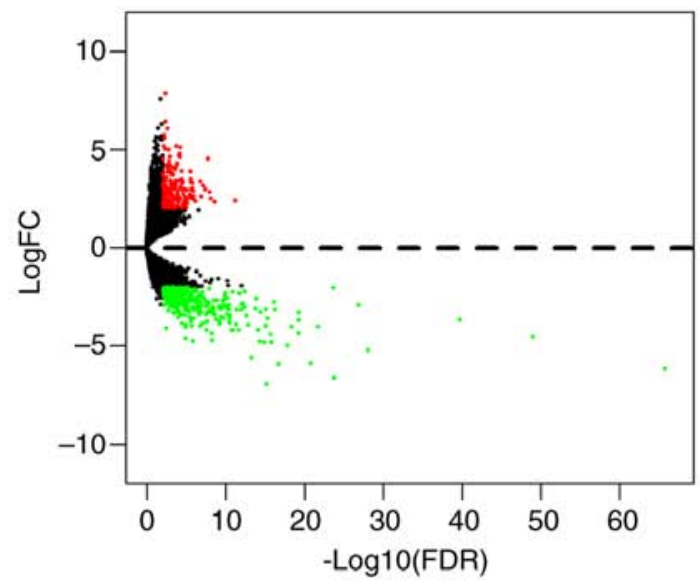

B

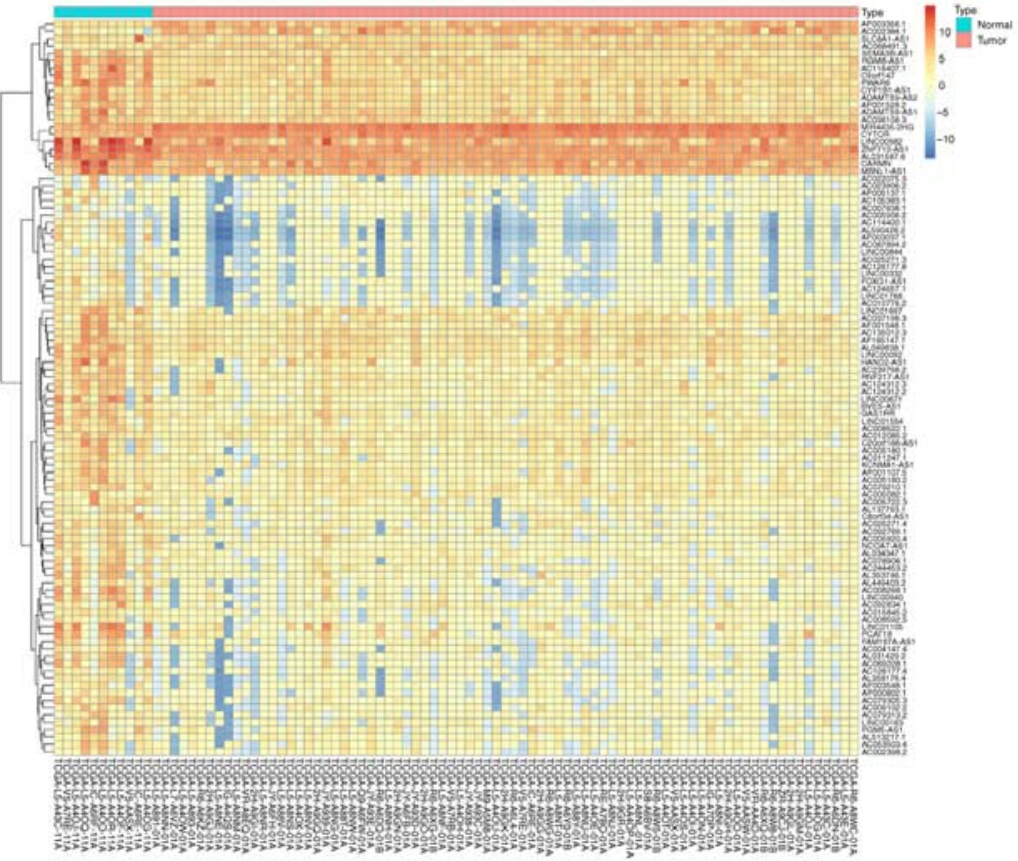

Figure 1. Volcano plot and heat map of lncRNA analysis. (A) Plots of $\log 2 \mathrm{FC}$ vs. $\log _{10}(\mathrm{FDR})$ for DElncRNAs. Red dots represent significantly upregulated lncRNAs and green dots represent significantly downregulated lncRNAs. (B) The top 100 significantly DElncRNAs were visualized by heat map. Each column represents one sample and each row represents a single lncRNA. DElncRNAs, differentially expressed lncRNAs; FC, fold change; FDR, false discovery rate; lncRNA, long non-coding RNA.

for differential gene expression analysis. For all P-values, a false discovery rate (FDR) was applied to correct the statistical significance of multiple testing. Genes with $>2.0$ fold change (FC) and FDR-adjusted $\mathrm{P}<0.01$ were considered significant. The volcano plot and heat map were designed to visualize the results using ggplots v3.0, which is a package based on the $\mathrm{R}$ language.

Association between DElncRNAs and patient prognosis. All patients were classified into high or low lncRNA-expression groups according to the median. Kaplan-Meier and log-rank methods were used to test differences between the two groups. $\mathrm{P}<0.05$ was considered to indicate a statistically significant difference.

Construction of the ceRNA network. Three calculations were performed to construct the ceRNA network as follows: i) Cancer-associated lncRNA filtration, where lncRNAs with FC>2.0 (either up- or downregulated) and FDR-adjusted $\mathrm{P}<0.01$ were considered as cancer-associated lncRNAs [to improve data reliability, cancer-associated lncRNAs that were not annotated by GENCODE (http://www.gencodegenes.org/) were excluded]; ii) lncRNA-miRNA interactions were predicted by miRcode (http://www.mircode.org/) and starBase (http://starbase.sysu.edu.cn/); and iii) target mRNAs of DEmiRNAs were predicted using the three bioinformatics databases miRDB (http://mirdb.org/), miRTarBase (http://mirtarbase.mbc.nctu.edu.tw/php/index.php) and TargetScan (http://www.targetscan.org). Gene Oncology (GO) was analyzed using Database for Annotation, Visualization, and Integrated Discovery bioinformatics tools (DAVID; v6.8; https://david.ncifcrf.gov). In order to improve the consistency of the bioinformatics analysis, the target genes were retained.
A network graph was constructed and visualized using Cytoscape v3.5.1 (https://cytoscape.org/).

\section{Results}

Patient characteristics. The detailed clinical information and pathological characteristics of the patients included in the present study, including sex, age at diagnosis, metastasis status, lymph node status and tumor-node-metastasis stage, are presented in Table I. The median age for all patients was 69 years (range, $27-86$ years). The median overall survival was 14.29 months (range 0.36-83.18 months).

Identification of DElncRNAs. IncRNAs with FDR-adjusted $\mathrm{P}<0.01$ and $\mathrm{FC}>2.0$ were considered to be differentially expressed. A total of 561 DElncRNAs were identified, of which 217 were upregulated and 344 were downregulated (Table SI). A volcano plot was therefore constructed (Fig. 1A) to visually describe the FDRs and FCs. In addition, a heat map was designed (Fig. 1B) to highlight the top 100 significant DElncRNAs according to the FDR-adjusted P-values.

To explore the potential lncRNAs that could possess prognostic abilities, the expression profiles of 561 lncRNAs and corresponding clinical data were analyzed using Kaplan-Meier Curve. The results demonstrated that 26 lncRNAs were positively correlated with overall survival (OS; P<0.05; Fig. 2). Among these 26, 12 lncRNAs, AC007128.1, AC079354.3, AC246680.1, AL009178.2, AL135924.2, AL138789.1, AP003356.1, AP0033469.2, GK-IT1, HOTAIR, LINC01114 and LINC01768, were negatively correlated with OS (Fig. 2A). Conversely, the remaining 14 lncRNAs, AC004585.1, AC016395.1, AC024337.2, AC087491.1, AC093583.1, AC104211.1, AL022316.1, AL031429.1, CYP1B1-AS1, 
A
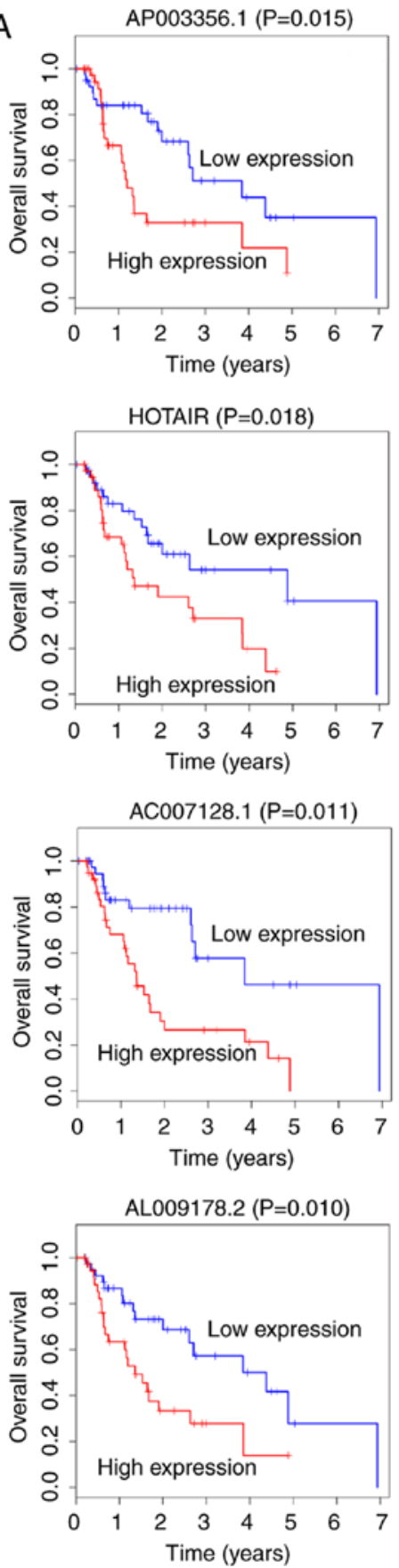
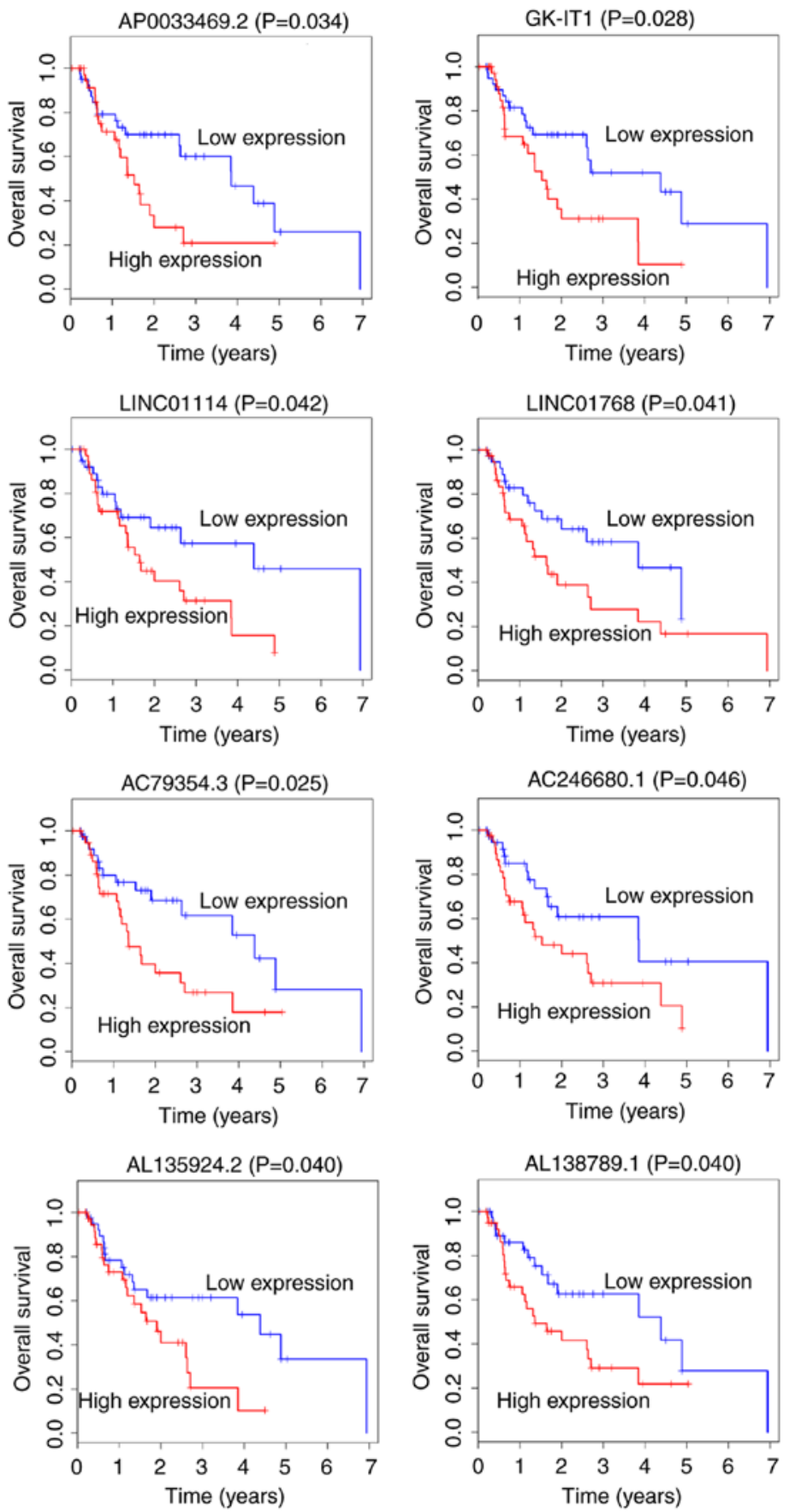

Figure 2. Kaplan-Meier survival curves for lncRNAs associated with OS ( $\mathrm{P}<0.05)$. (A) A total of 12 lncRNAs were negatively correlated with OS. lncRNA, long non-coding RNA; OS, overall survival.

LINC00163, LINC00906, LINC01695, SLCO4A1-AS1 and UG0898H09, were positively correlated with OS (Fig. 2B).

Identification of DEmRNAs. The RNA expression levels in 79 EAC tumor samples and 11 normal samples were analyzed. With a cut-off value of FDR-adjusted $\mathrm{P}<0.01$ and $\mathrm{FC}>2.0,367$ upregulated and 922 downregulated mRNAs were identified (Table SII). A volcano plot was therefore constructed to visualize the results (Fig. 3A). The top 100 significant DEmRNAs were then highlighted by plotting FDR-adjusted P-values in a heat map (Fig. 3C).

To analyze the DEmRNA functions, enrichment analysis based on enriched functional GO modules was performed.
The results demonstrated that the DEmRNAs were significantly enriched in the 'chemokine-mediated signaling pathway' (GO: 0070098), 'plasma membrane' (GO: 0005886) and 'calcium ion binding' (GO: 0005509) GO terms under 'biological process', 'cellular component' and 'molecular function', respectively (Fig. 3B).

Identification of DEmiRNAs. In order to design a ceRNA network for EAC, the miRNA expression profiles between tumor samples and normal samples were compared. Subsequently, 44 DEmiRNAs, including 28 upregulated and 16 downregulated were identified (Fig. 4A and C). The mRNAs that were targeted by the 44 DEmiRNAs from miRDB, 
B
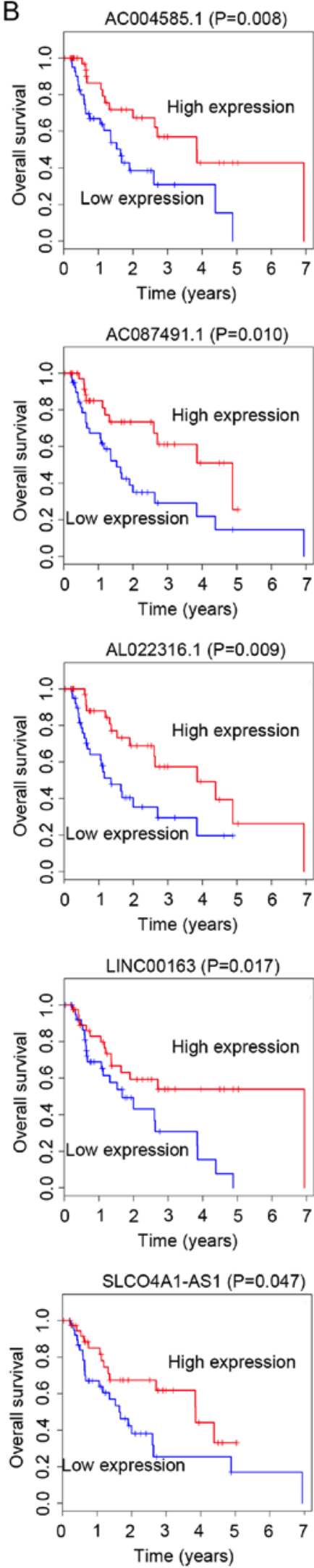
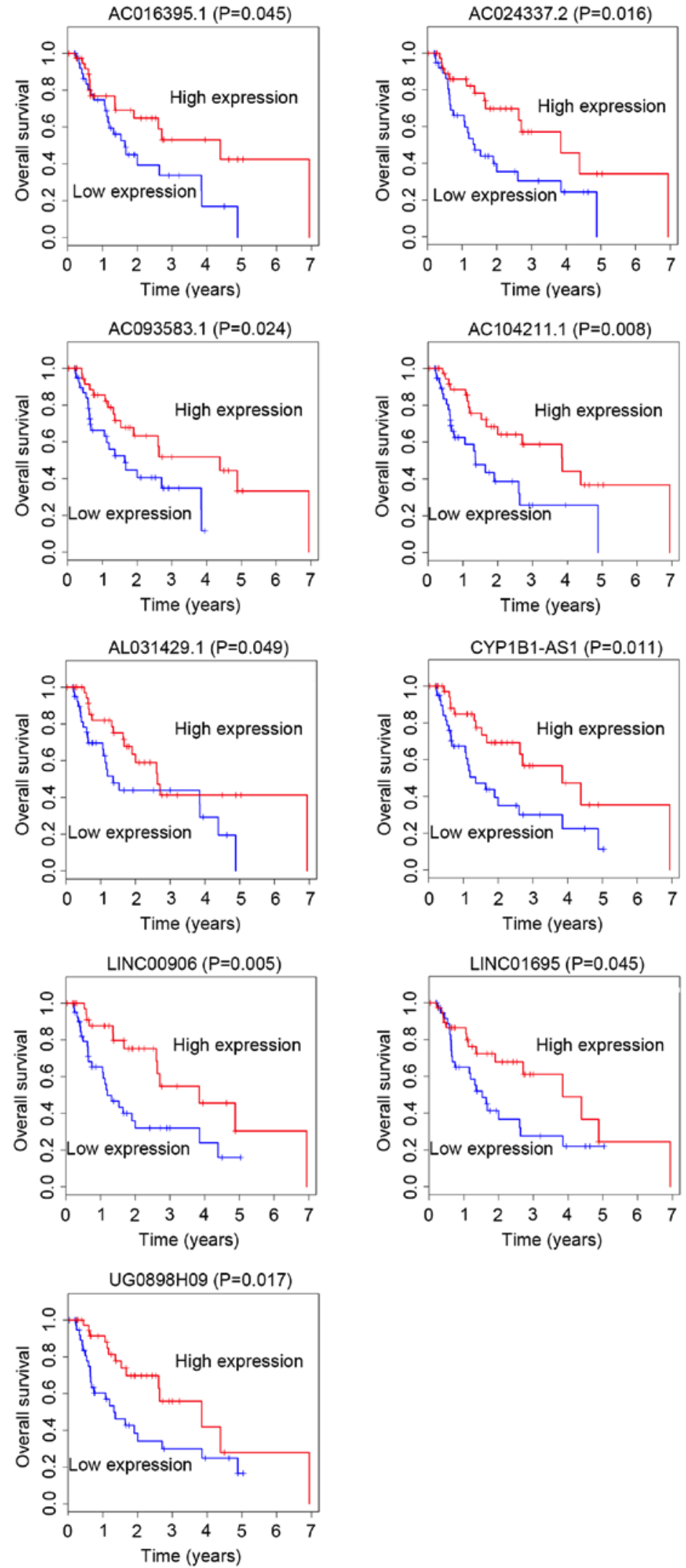

Figure 2. Continued. (B) A total of 14 lncRNAs were positively correlated with overall survival. lncRNA, long non-coding RNA; OS, overall survival.

miRTarBase and TargetScan were then screened. To improve data reliability, mRNAs that were not included in the set of 1,289 DEmRNAs were excluded. Eventually, 13 DEmiRNAs remained in the ceRNA network (Fig. 4B).
In order to identify the DEmRNAs that may have potential prognostic ability, the expression profiles of the 13 DEmRNAs included in the ceRNA network were analyzed using Kaplan-Meier curve. The results demonstrated that the 

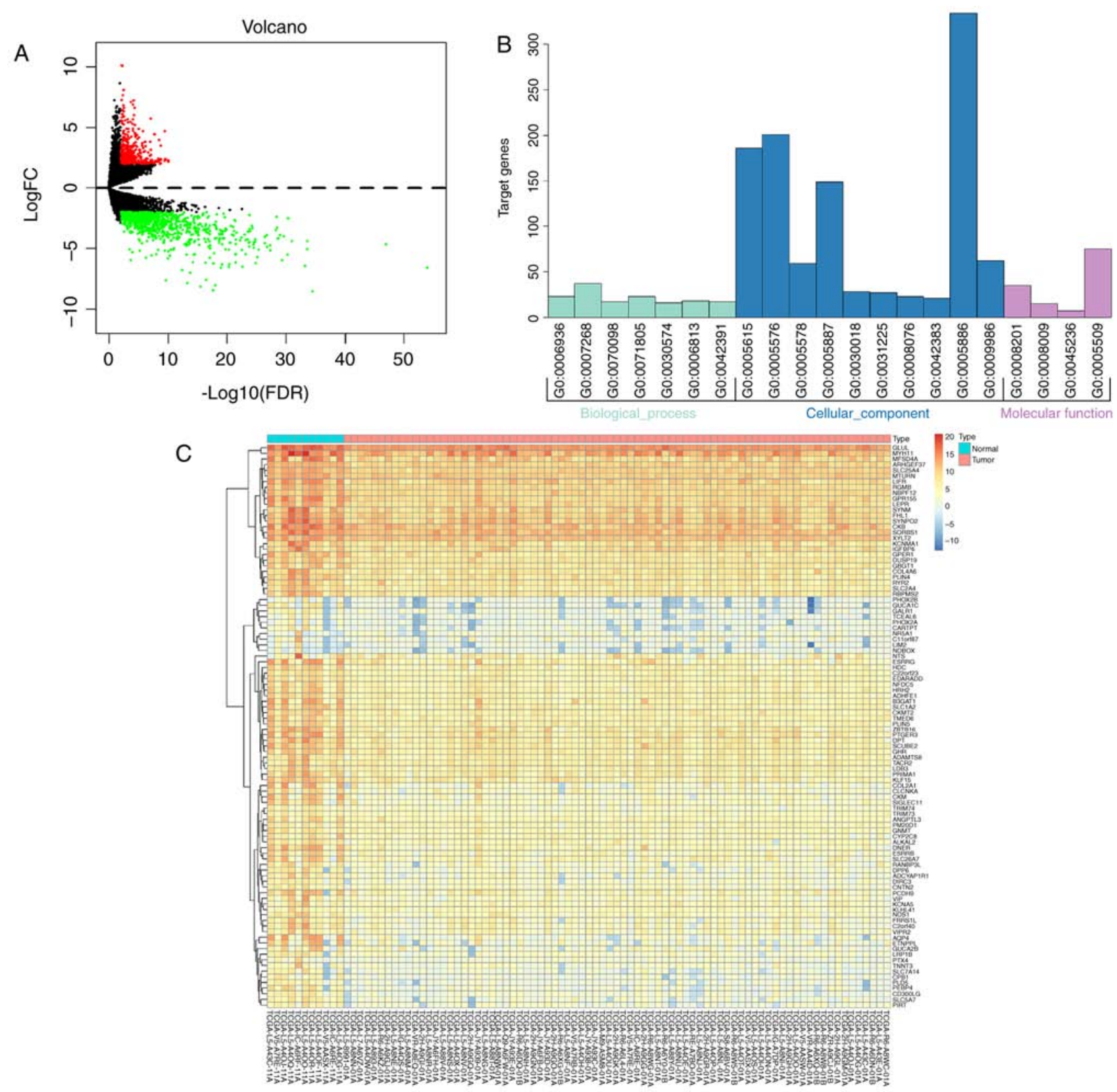

Figure 3. DEmRNAs in esophageal adenocarcinoma. (A) Volcano plot of $\log 2 \mathrm{FC}$ vs. $\log _{10}$ (FDR) for DEmRNAs. Red dots represent significantly upregulated mRNAs, and green dots represent significantly downregulated mRNAs. (B) The enriched Gene Ontology terms of target genes. (C) The top 100 significant DEmRNAs were visualized by heat map. Each column represents one sample and each row represents a single mRNA. DEmRNAs, differentially expressed mRNAs; FC, fold change; FDR, false discovery rate.

expression profiles of three DEmRNAs were positively correlated with OS $(\mathrm{P}<0.05)$. Two of these DEmRNAs, angiopoietin 2 and interleukin 11 (IL11) were negatively correlated with OS, whereas neurotrophic receptor tyrosine kinase 2 (NTRK2) was positively correlated with OS (Fig. 4D).

To identify the IncRNA-miRNA interactions in EAC, the potential MREs in DElncRNAs were screened using miRcode. For miRNA-mRNA interactions, miRDB, miRTarBase, and TargetScan were used to identify the DEmRNAs targeted by DEmiRNAs. The results are listed in Tables II and III.

ceRNA network construction. In order to improve knowledge on DElncRNA function in EAC, a dysregulated IncRNA-miRNA-mRNA ceRNA network based on the aforementioned data (presented in Tables II and III) was constructed. The results demonstrated that in the ceRNA network, interaction of five DEmiRNAs with 37 DElncRNAs was predicted, according to the results retrieved from miRcode. The ceRNA network is presented in Fig 5.

\section{Discussion}

lncRNAs represent a crucial category of non-coding genes in the transcriptome that act as pivotal regulators of cell physiology and pathology in human cancer by mediating gene expression through multiple mechanisms (28). The dysregulation of IncRNA expression is involved in the pathogenesis of various types of solid tumor $(29,30)$. Numerous novel biological 

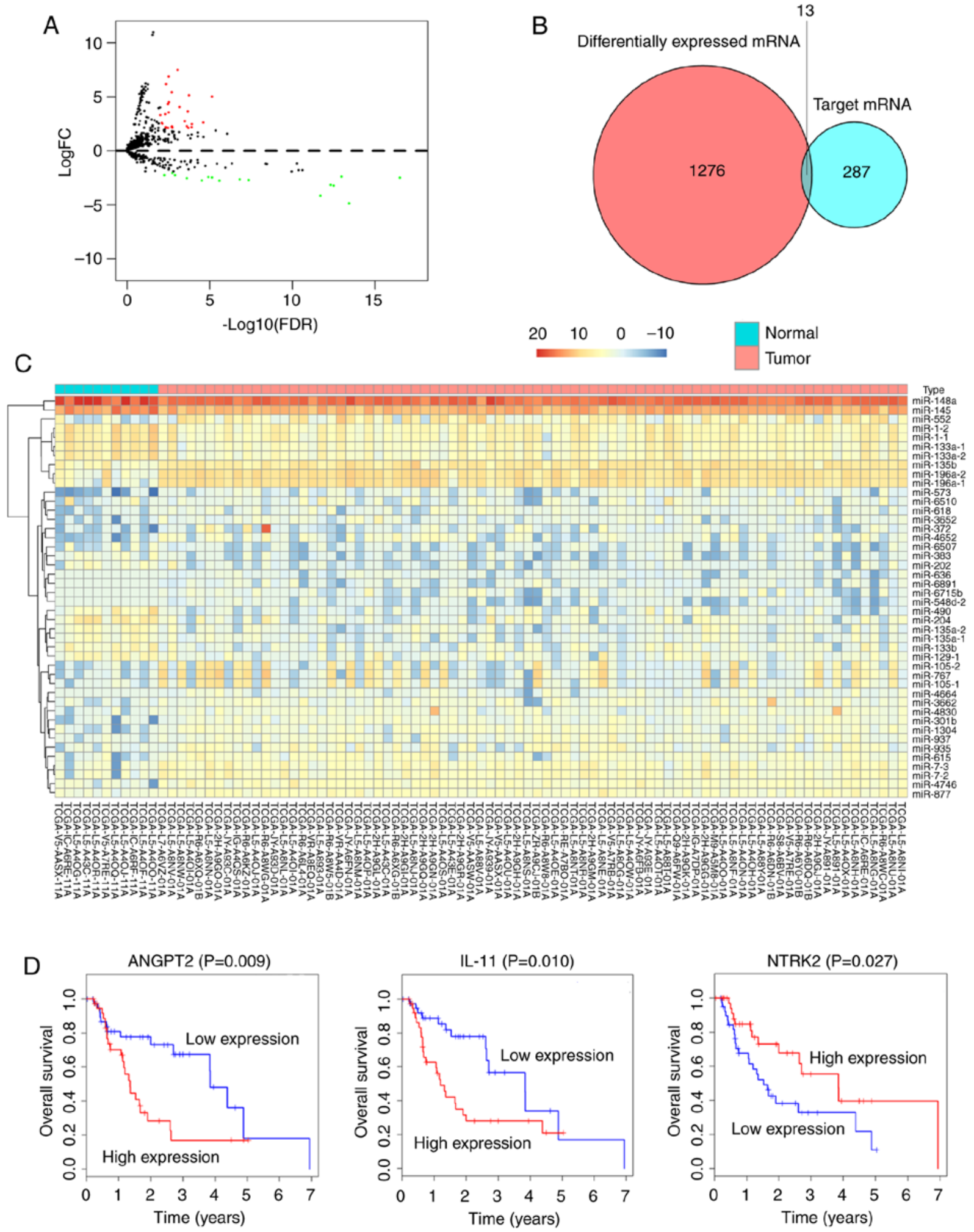

Figure 4. DEmiRNAs in esophageal adenocarcinoma. (A) Volcano plot of $\log 2 \mathrm{FC}$ vs. $\log _{10}$ (FDR) for differentially expressed miRNAs. Red dots represent significantly upregulated miRNAs and green dots represent significantly downregulated miRNAs. (B) Venn analysis of overlapping genes between statistically significant mRNAs and target genes of DEmiRNAs. (C) Significant DEmiRNAs were visualized by heat map. Each column represents one sample and each row represents an individual miRNA. (D) Kaplan-Meier survival curves of three target genes in overall survival prediction (P<0.05). DEmiRNAs, differentially expressed miRNAs; FC, fold change; FDR, false discovery rate; ANGPT2, angiopoietin 2; IL-11, interleukin-11; NTRK2, neurotrophic receptor tyrosine kinase 2; miRNA, microRNA.

functions have been attributed to lncRNAs, which have become the focal point of many studies $(10,17)$. The ceRNA hypothesis describes regulatory networks among protein-coding mRNAs and non-coding RNAs, including miRNAs and lncRNAs at the post-transcription level. According to this hypothesis, changes in the expression of one or multiple miRNA targets can alter the number of unbound miRNAs and lead to observable changes in miRNA activity. The various transcripts 


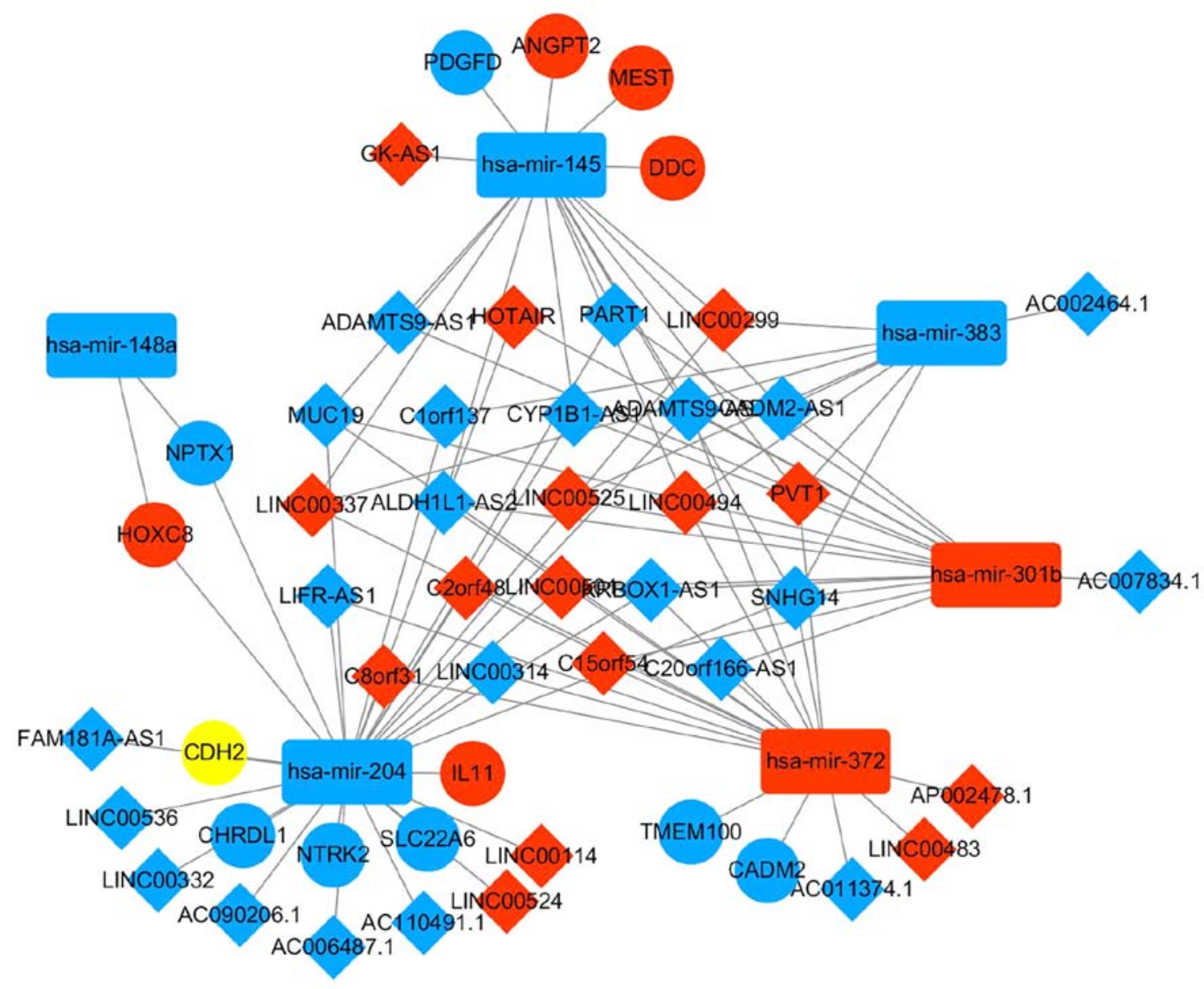

Figure 5. Differentially expressed lncRNA-mediated competing endogenous RNA network in EAC. Global view of the lncRNA-miRNA-mRNA network in EAC. The nodes highlighted in red indicate an increased level of expression and the nodes highlighted in blue indicate a decreased level of expression. lncRNAs, miRNAs, and mRNAs are indicated as diamonds, rounded rectangles and ellipses, respectively. EAC, esophageal adenocarcinoma; lncRNA, long non-coding RNA; miRNA/miR, microRNA.

from the transcriptome communicate with one another by competitively binding to shared MREs (20). ceRNA networks in human cancer include cancer-associated lncRNAs, microRNAs and mRNAs. A previous study demonstrated a miRNA-lncRNA-mRNA interaction in ESCC (31). However, the ceRNA network in EAC remains poorly understood.

At present, since lncRNAs are able to regulate miRNA functions by competitively binding to shared MREs in mRNA, they are considered as diagnostic and prognostic biomarkers. Numerous well-studied lncRNAs have been identified as potential targets or powerful predictors in various types of cancer, including LINC00668, H19 and UCA1 (32-34). However, studies on EAC remain rare. Based on the RNA-Seq data and clinical data from 79 patients with EAC, the present study demonstrated that 26 cancer-associated lncRNAs may affect the OS of patients with EAC. In particular, the results from this study reported that two DElncRNAs, CYP1B1-AS1 and HOTAIR, were not only identified as part of the ceRNA network, but were also positively and negatively correlated with OS, respectively, which suggested that these two lncRNAs may serve as essential oncogenes and as prognostic markers in EAC.

HOTAIR is a highly studied lncRNA. Previous studies demonstrated that it serves a role in the development and progression of various types of solid tumor, including renal cell carcinoma, colorectal cancer, breast cancer, gastric cancer, non-small cell lung cancer, cervical cancer and ovarian epithelial carcinoma (35-45). In addition, Ren et al (46) demonstrated that HOTAIR can control the cell cycle by acting as a competing endogenous 'sponge' to downregulate miR-1 and upregulate cyclin D1 in ESCC. The present study reported that HOTAIR expression was upregulated in EAC tumor tissues. In addition, patients with highly expressed HOTAIR had worse survival outcomes. HOTAIR may therefore compete with miR-301b and miR-204 to regulate chordin like 1, NTRK2, IL11, neuronal pentraxin 1, homeobox C8 and solute carrier family 22 member 6 expression. Although these mRNAs have been identified as aberrantly expressed, their roles have not been fully investigated in EAC.

In addition to HOTAIR, LINC00163 and SLCO4A1-AS1 have also been reported to be associated with cancer prognosis. Guo et al (47) demonstrated that the LINC00163 level is significantly decreased in lung cancer tissues and cell lines following bioinformatics and reverse transcription-quantitative PCR analyses. LINC00163 expression was lower in metastatic tissues compared with non-metastatic tissues, and a higher LINC00163 expression in patients with lung cancer could predict a better prognosis. Yang et al (48) reported that SLCO4A1-AS1 expression was more upregulated in bladder cancer tissues compared with that in adjacent normal tissues, and that SLCO4A1-AS1 overexpression is associated with poor 
Table II. Potential miRNAs that may target cancer-associated lncRNAs by miRNA response elements.

\begin{tabular}{|c|c|}
\hline lncRNAs & miRNAs \\
\hline LINC00525 & hsa-mir-301b, hsa-mir-383 \\
\hline PART1 & hsa-mir-301b, hsa-mir-145, hsa-mir-204 \\
\hline C2orf48 & hsa-mir-372, hsa-mir-372 \\
\hline LINC00483 & hsa-mir-372 \\
\hline C20orf166-AS1 & hsa-mir-301b, hsa-mir-372 \\
\hline C15orf54 & hsa-mir-301b, hsa-mir-372 \\
\hline C8orf31 & hsa-mir-372, hsa-mir-204 \\
\hline AP002478.1 & hsa-mir-372 \\
\hline LINC00314 & hsa-mir-372, hsa-mir-204 \\
\hline LINC00501 & hsa-mir-301b, hsa-mir-204 \\
\hline C1orf137 & hsa-mir-204, hsa-mir-383 \\
\hline MUC19 & $\begin{array}{l}\text { hsa-mir-301b, hsa-mir-372, hsa-mir-145, } \\
\text { hsa-mir-204 }\end{array}$ \\
\hline KRBOX1-AS1 & hsa-mir-301b, hsa-mir-372, hsa-mir-204 \\
\hline LINC00114 & hsa-mir-204 \\
\hline SNHG14 & $\begin{array}{l}\text { hsa-mir-301b, hsa-mir-372, hsa-mir-145, } \\
\text { hsa-mir-204 }\end{array}$ \\
\hline LINC00337 & hsa-mir-372, hsa-mir-145, hsa-mir-383 \\
\hline HOTAIR & hsa-mir-301b, hsa-mir-204 \\
\hline LINC00332 & hsa-mir-204 \\
\hline CYP1B1-AS1 & hsa-mir-301b, hsa-mir-145, hsa-mir-204 \\
\hline AC110491.1 & hsa-mir-204 \\
\hline LINC00494 & hsa-mir-372, hsa-mir-145, hsa-mir-383 \\
\hline LINC00299 & hsa-mir-145, hsa-mir-204, hsa-mir-383 \\
\hline CADM2-AS1 & hsa-mir-301b, hsa-mir-145, hsa-mir-383 \\
\hline AC011374.1 & hsa-mir-372 \\
\hline ADAMTS9-AS1 & hsa-mir-301b, hsa-mir-145 \\
\hline AC002464.1 & hsa-mir-383 \\
\hline AC007834.1 & hsa-mir-301b \\
\hline ADAMTS9-AS2 & $\begin{array}{l}\text { hsa-mir-301b, hsa-mir-372, hsa-mir-145, } \\
\text { hsa-mir-204 }\end{array}$ \\
\hline GK-AS1 & hsa-mir-145 \\
\hline LIFR-AS1 & hsa-mir-372, hsa-mir-204 \\
\hline ALDH1L1-AS2 & hsa-mir-301b, hsa-mir-372, hsa-mir-145 \\
\hline PVT1 & hsa-mir-372, hsa-mir-145, hsa-mir-383 \\
\hline LINC00536 & hsa-mir-204 \\
\hline AC006487.1 & hsa-mir-204 \\
\hline AC090206.1 & hsa-mir-204 \\
\hline FAM181A-AS1 & hsa-mir-204 \\
\hline LINC00524 & hsa-mir-204 \\
\hline
\end{tabular}

lncRNA, long non-coding RNA; miRNA, microRNA.

prognosis and tumor metastasis. Yu et al (49) demonstrated that a high SLCO4A1-AS1 expression level is associated with bladder cancer progression and that SLCO4A1-AS1 promotes malignant phenotypes of bladder cancer cells via the miRNA-335-5p/OCT4 axis.

To confirm the accuracy of the ceRNA network prediction presented in this study, interactions among lncRNAs,
Table III. Differentially expressed mRNAs targeted by differentially expressed miRNAs.

\begin{tabular}{ll}
\hline miRNAs & \multicolumn{1}{c}{ mRNAs } \\
\hline hsa-mir-145 & ANGPT2, PDGFD, DDC, MEST \\
hsa-mir-148a & HOXC8, NPTX1 \\
hsa-mir-196b & HOXB7, IGF2BP3, HOXC8 \\
hsa-mir-204 & HOXC8, NTRK2, SLC22A6, CHRDL1, \\
& IL11, NPTX1, CDH2 \\
hsa-mir-372 & TMEM100, CADM2
\end{tabular}

miRNA, microRNA.

miRNAs and mRNAs in EAC were measured. Only cancer-associated lncRNAs and miRNAs with $>2.0 \mathrm{FC}$ and FDR $<0.01$ were selected. These non-coding genes were then annotated by GENCODE. Interactions among lncRNAs, miRNAs and mRNAs were predicted by experimentally conformed algorithms or by using miRDB, miRcode, miRTarBase and TargetScan databases. In the present study, cancer-associated lncRNAs in EAC were identified based on the RNA-Seq data of 79 EAC tissues and 11 normal tissues. Subsequently, cancer-associated miRNAs and mRNAs were identified. Eventually, interactions between lncRNAs, miRNAs and mRNAs was identified by constructing an lncRNA-miRNA-mRNA ceRNA network. A total of 37 DElncRNAs, five miRNAs and 13 mRNAs were selected to construct this newly-identified ceRNA-mediated gene regulatory network. This network included numerous active lncRNA-miRNA-mRNA interactions that may be used as prognostic biomarkers in EAC.

In conclusion, the present study identified some cancer-associated lncRNAs and revealed their potential use in prognosis prediction. In particular, some cancer-associated lncRNAs may serve as ceRNAs. The ceRNA network that was built in the present study may help understanding the mechanisms involved in the development and progression of EAC.

\section{Acknowledgements}

Not applicable.

\section{Funding}

No funding was received.

\section{Availability of data and materials}

The datasets used and/or analyzed during the current study are available from the corresponding author on reasonable request.

\section{Authors' contributions}

SC and YY conceived and designed the study. XC acquired the data. YY analyzed and interpreted the data. YY and XC wrote and revised the manuscript. SC supervised the study. All authors read and approved the final manuscript. 


\section{Ethics approval and consent to participate}

Not applicable.

\section{Patient consent for publication}

Not applicable.

\section{Competing interests}

The authors declare that they have no competing interests.

\section{References}

1. Mannath J and Ragunath K: Role of endoscopy in early oesophageal cancer. Nat Rev Gastroenterol Hepatol 13: 720-730, 2016.

2. Siegel RL, Miller KD and Jemal A: Cancer statistics, 2016. CA Cancer J Clin 66: 7-30, 2016.

3. Chen W, Zheng R, Baade PD, Zhang S, Zeng H, Bray F, Jemal A Yu XQ and He J: Cancer statistics in China, 2015. CA Cancer J Clin 66: 115-132, 2016.

4. Pera M, Manterola C, Vidal O and Grande L: Epidemiology of esophageal adenocarcinoma. J Surg Oncol 92: 151-159, 2005

5. Coleman HG, Xie SH and Lagergren J: The epidemiology of esophageal adenocarcinoma. Gastroenterology 154: 390-405, 2018.

6. Huang X, Zhou X, Hu Q, Sun B, Deng M, Qi X and Lü M Advances in esophageal cancer: A new perspective on pathogenesis associated with long non-coding RNAs. Cancer Lett 413: 94-101, 2018

7. Lagergren $J$ and Lagergren P: Recent developments in esophageal adenocarcinoma. CA Cancer J Clin 63: 232-248, 2013.

8. Rustgi AK and El-Serag HB: Esophageal carcinoma. N Engl J Med 371: 2499-2509, 2014.

9. Schmitt AM and Chang HY: Long noncoding RNAs in cancer pathways. Cancer Cell 29: 452-463, 2016.

10. Chen JF, Wu P, Xia R, Yang J, Huo XY, Gu DY, Tang CJ, De W and Yang F: STAT3-induced lncRNA HAGLROS overexpression contributes to the malignant progression of gastric cancer cells via mTOR signal-mediated inhibition of autophagy. Mol Cancer 17: 6, 2018.

11. Zhou DD, Liu XF, Lu CW, Pant OP and Liu XD: Long non-coding RNA PVT1: Emerging biomarker in digestive system cancer. Cell Prolif 50, 2017.

12. Song YX, Sun JX, Zhao JH, Yang YC, Shi JX, Wu ZH, Chen XW, Gao P, Miao ZF and Wang ZN: Non-coding RNAs participate in the regulatory network of CLDN4 via ceRNA mediated miRNA evasion. Nat Commun 8: 289, 2017.

13. Parasramka MA, Maji S, Matsuda A, Yan IK and Patel T: Long non-coding RNAs as novel targets for therapy in hepatocellular carcinoma. Pharmacol Ther 161: 67-78, 2016.

14. Li W, Zheng J, Deng J, You Y, Wu H, Li N, Lu J and Zhou Y: Increased levels of the long intergenic non-protein coding RNA POU3F3 promote DNA methylation in esophageal squamous cell carcinoma cells. Gastroenterology 146: 1714-1726.e5, 2014.

15. Xiao ZD, Han L, Lee H, Zhuang L, Zhang Y, Baddour J, Nagrath D, Wood CG, Gu J, Wu X, et al: Energy stress-induced lncRNA FILNC1 represses c-Myc-mediated energy metabolism and inhibits renal tumor development. Nat Commun 8: 783, 2017.

16. Martens-Uzunova ES, Böttcher R, Croce CM, Jenster G, Visakorpi T and Calin GA: Long noncoding RNA in prostate, bladder, and kidney cancer. Eur Urol 65: 1140-1151, 2014.

17. Wang $\mathrm{P}, \mathrm{Xu}$ J, Wang $\mathrm{Y}$ and Cao X: An interferon-independent IncRNA promotes viral replication by modulating cellular metabolism. Science 358: 1051-1055, 2017.

18. Su W, Guo C, Wang L, Wang Z, Yang X, Niu F, Tzou D, Yang X Huang X, Wu J, et al: LncRNA MIR22HG abrogation inhibits proliferation and induces apoptosis in esophageal adenocarcinoma cells via activation of the STAT3/c-Myc/FAK signaling. Aging (Albany NY) 11: 4587-4596, 2019.

19. Salmena L, Poliseno L, Tay Y, Kats L and Pandolfi PP: A ceRNA hypothesis: The rosetta stone of a hidden RNA language. Cell 146: 353-358, 2011.

20. Qi X, Zhang DH, Wu N, Xiao JH, Wang X and Ma W: ceRNA in cancer: Possible functions and clinical implications. J Med Genet 52: 710-718, 2015 .
21. Cesana M, Cacchiarelli D, Legnini I, Santini T, Sthandier O, Chinappi M, Tramontano A and Bozzoni I: A long noncoding RNA controls muscle differentiation by functioning as a competing endogenous RNA. Cell 147: 358-369, 2011.

22. Qu L, Ding J, Chen C, Wu ZJ, Liu B, Gao Y, Chen W, Liu F, Sun W, Li XF, et al: Exosome-transmitted lncARSR promotes Sunitinib resistance in renal cancer by acting as a competing endogenous RNA. Cancer Cell 29: 653-668, 2016.

23. Cao C, Zhang T, Zhang D, Xie L, Zou X, Lei L, Wu D and Liu L: The long non-coding RNA, SNHG6-003, functions as a competing endogenous RNA to promote the progression of hepatocellular carcinoma. Oncogene 36: 1112-1122, 2017.

24. Conte F, Fiscon G, Chiara M, Colombo T, Farina L and Paci P: Role of the long non-coding RNA PVT1 in the dysregulation of the ceRNA-ceRNA network in human breast cancer. PLoS One 12: e0171661, 2017

25. Yang S, Ning Q, Zhang G, Sun H, Wang Z and Li Y: Construction of differential mRNA-lncRNA crosstalk networks based on ceRNA hypothesis uncover key roles of lncRNAs implicated in esophageal squamous cell carcinoma. Oncotarget 7: 85728-85740, 2016.

26. Li CY, Liang GY, Yao WZ, Sui J, Shen X, Zhang YQ, Peng H, Hong WW, Ye YC, Zhang ZY, et al: Integrated analysis of long non-coding RNA competing interactions reveals the potential role in progression of human gastric cancer. Int J Oncol 48: 1965-1976, 2016.

27. Team RC: R: A language and environment for statistical computing, R Foundation for Statistical Computing, Vienna, Austria, 2013

28. Marchese FP, Raimondi I and Huarte M: The multidimensional mechanisms of long noncoding RNA function. Genome Biol 18: 206, 2017.

29. Alvarez-Dominguez JR and Lodish HF: Emerging mechanisms of long noncoding RNA function during normal and malignant hematopoiesis. Blood 130: 1965-1975, 2017.

30. Klingenberg M, Matsuda A,Diederichs S and Patel T: Non-coding RNA in hepatocellular carcinoma: Mechanisms, biomarkers and therapeutic targets. J Hepatol 67: 603-618, 2017.

31. Lin C, Zhang N, Wang Y, Wang Y, Nice E, Guo C, Zhang E, Yu L, $\mathrm{Li}$ M,Liu C, et al: Functional role of a novel long noncoding RNA TTN-AS1s in esophageal squamous cell carcinoma progression and metastasis. Clin Cancer Res 24: 486-498, 2018.

32. Zhang E, Yin D, Han L, He X, Si X, Chen W, Xia R, Xu T, Gu D, De W, et al: E2F1-induced upregulation of long noncoding RNA LINC00668 predicts a poor prognosis of gastric cancer and promotes cell proliferation through epigenetically silencing of CKIs. Oncotarget 7: 23212-23226, 2016.

33. Liu L, Liu L and Lu S: IncRNA H19 promotes viability and epithelial-mesenchymal transition of lung adenocarcinoma cells by targeting miR-29b-3p and modifying STAT3. Int J Oncol 54: 929-941, 2019.

34. Cui X, Zhao C, Yao X, Qian B, Su C, Ren Y, Yao Z, Gao X and Yang J: SND1 acts as an anti-apoptotic factor via regulating the expression of IncRNA UCA1 in hepatocellular carcinoma. RNA Biol 15: 1364-1375, 2018

35. Katayama H, Tamai K, Shibuya R, Nakamura M, Mochizuki M, Yamaguchi K, Kawamura S, Tochigi T, Sato I, Okanishi T, et al: Long non-coding RNA HOTAIR promotes cell migration by upregulating insulin growth factor-binding protein 2 in renal cell carcinoma. Sci Rep 7: 12016, 2017

36. Li M, Li X, Zhuang Y, Flemington EK, Lin Z and Shan B: Induction of a novel isoform of the lncRNA HOTAIR in Claudin-low breast cancer cells attached to extracellular matrix. Mol Oncol 11: 1698-1710, 2017.

37. Li P, Zhang X, Wang L, Du L, Yang Y, Liu T, Li C and Wang C: lncRNA HOTAIR contributes to 5FU resistance through suppressing miR-218 and activating NF- $\mathrm{B} / \mathrm{TS}$ signaling in colorectal cancer. Mol Ther Nucleic Acids 8: 356-369, 2017

38. Ma J, Fan Y, Feng T, Chen F, Xu Z, Li S, Lin Q, He X, Shi W, Liu Y, et al: HOTAIR regulates HK2 expression by binding endogenous miR-125 and miR-143 in oesophageal squamous cell carcinoma progression. Oncotarget 8: 86410-86422, 2017.

39. Chi S, Shen L, Hua T, Liu S, Zhuang G, Wang X, Zhou X, Wang $\mathrm{G}$ and Wang $\mathrm{H}$ : Prognostic and diagnostic significance of lncRNAs expression in cervical cancer: A systematic review and meta-analysis. Oncotarget 8: 79061-79072, 2017.

40. Fan CN, Ma L and Liu N: Systematic analysis of lncRNA-miRNA-mRNA competing endogenous RNA network identifies four-lncRNA signature as a prognostic biomarker for breast cancer. J Transl Med 16: 264, 2018. 
41. Gao S, Zhao ZY, Wu R, Zhang Y and Zhang ZY: Prognostic value of long noncoding RNAs in gastric cancer: A meta-analysis. OncoTargets Ther 11: 4877-4891, 2018.

42. Guo F, Cao Z, Guo H and Li S: The action mechanism of IncRNA-HOTAIR on the drug resistance of non-small cell lung cancer by regulating Wnt signaling pathway. Exp Ther Med 15: 4885-4889, 2018.

43. Qiu JJ, Lin YY, Ye LC, Ding JX, Feng WW, Jin HY, Zhang Y, $\mathrm{Li} \mathrm{Q}$ and Hua KQ: Overexpression of long non-coding RNA HOTAIR predicts poor patient prognosis and promotes tumor metastasis in epithelial ovarian cancer. Gynecol Oncol 134: 121-128, 2014.

44. Wang WJ, Li HT, Yu JP, Han XP, Xu ZP, Li YM, Jiao ZY and Liu HB: A competing endogenous RNA network reveals novel potential lncRNA, miRNA, and mRNA biomarkers in the prognosis of human colon adenocarcinoma. J Surg Res 235: 22-33, 2019.

45. Zhang H, Wang Z, Wu J, Ma R and Feng J: Long noncoding RNAs predict the survival of patients with colorectal cancer as revealed by constructing an endogenous RNA network using bioinformation analysis. Cancer Med 8: 863-873, 2019.
46. Ren K, Li Y, Lu H, Li Z, Li Z, Wu K, Li Z and Han X: Long noncoding RNA HOTAIR controls cell cycle by functioning as a competing endogenous RNA in esophageal squamous cell carcinoma. Transl Oncol 9: 489-497, 2016.

47. Guo X, Wei Y, Wang Z, Liu W, Yang Y, Yu X and He J: LncRNA LINC00163 upregulation suppresses lung cancer development though transcriptionally increasing TCF21 expression. Am J Cancer Res 8: 2494-2506, 2018.

48. Yang Y, Wang F, Huang H, Zhang Y, Xie H and Men T: lncRNA SLCO4A1-AS1 promotes growth and invasion of bladder cancer through sponging miR-335-5p to upregulate OCT4. OncoTargets Ther 12: 1351-1358, 2019.

49. Yu J, Han Z, Sun Z, Wang Y, Zheng M and Song C: LncRNA SLCO4A1-AS1 facilitates growth and metastasis of colorectal cancer through $\beta$-catenin-dependent Wnt pathway. J Exp Clin Cancer Res 37: 222, 2018.

This work is licensed under a Creative Commons Attribution-NonCommercial-NoDerivatives 4.0 International (CC BY-NC-ND 4.0) License. 